Research Article

\title{
Bionics Design of Artificial Leg and Experimental Modeling Research of Pneumatic Artificial Muscles
}

\author{
Hualong Xie $\mathbb{D}^{1}{ }^{1}$ Zhijie Li $\mathbb{D}^{1},{ }^{1}$ and Fei $\mathrm{Li}^{2}$ \\ ${ }^{1}$ Institute of Advanced Manufacturing and Automation, School of Mechanical Engineering and Automation, \\ Northeastern University, Shenyang, China \\ ${ }^{2}$ School of Information Science \& Engineering, Shenyang University of Technology Shenyang, Shenyang, China \\ Correspondence should be addressed to Hualong Xie; hlxie@mail.neu.edu.cn
}

Received 19 November 2019; Accepted 8 January 2020; Published 28 February 2020

Academic Editor: L. Fortuna

Copyright (c) 2020 Hualong Xie et al. This is an open access article distributed under the Creative Commons Attribution License, which permits unrestricted use, distribution, and reproduction in any medium, provided the original work is properly cited.

\begin{abstract}
In the research and development of intelligent prosthesis, some of performance test experiments are required. In order to provide an ideal experimental platform for the performance test of intelligent prosthesis, a heterogeneous biped walking robot model is proposed. Artificial leg is an important part of heterogeneous biped walking robot, and its main function is to simulate the disabled a healthy normal gait, which provides intelligent bionic legs gait to follow the target trajectory. The pneumatic artificial muscles (PAM) have good application in the artificial leg. The bionic design of artificial leg mainly includes the structure of hip joint, knee joint, and ankle joint, adopting the four-bar mechanism as the mechanical structure of the knee joint, and PAM are used as the driving source of the knee joint. Secondly, the PAM performance test platform is built to establish the relationship among output force, shrinkage rate, and input pressure under the measured isobaric conditions, and the mathematical model of PAM is established. Finally, the virtual prototype technology is used to build a joint simulation platform, and PID control algorithm is used for verification simulation. The results show that the artificial leg can follow the target trajectory.
\end{abstract}

\section{Introduction}

In order to provide convenience for lower limb amputees, intelligent prosthetics are used to replace disabled limbs in the field of biological rehabilitation, and the development and research of intelligent prosthetics require ideal artificial legs [1]. Most two-legged robots use direct-current (DC) servo motors as driving devices, and some also use pneumatic and hydraulic devices. Although the position accuracy of hydraulic device driving can be guaranteed, its stiffness is large, and leakage and pollution may occur. Pneumatic device drive is mainly used for joint position control, and the requirement of sealing cylinder is high. As a new type of pneumatic component, PAM has the advantages of simple structure, good flexibility, large output force, light weight, and similar mechanical properties to biological muscle [2]. Therefore, pneumatic artificial muscle is adopted as the driving device of the knee joint, combining the research of humanoid robot and intelligent prosthesis to study the artificial leg in the walking robot mode of heterogeneous legs [3] and make it simulate the gait of healthy legs of disabled people.

Chou and Hannaford studied the PAM in detail from the biological point of view $[4,5]$. According to the principle of energy conservation, the static mathematical model of the pneumatic artificial muscle was inferred, and its static characteristics were tested. Van Damme et al. proposed a Preisach-based model to describe hysteresis in pleated PAM [6]. Zhong found that the parameters of Bouc-Wen model were optimized, and their relationships with pressure applied to PAM are discussed. Wang et al. proposed a cascade control strategy based on robust modeling for simple and efficient trajectory tracking control of a bionic joint driven by a single PAM $[7,8]$.

La Rosa et al. formed a single chain by inhibitive chemical synapse in modified version of the Hindmarsh-Rose model and discussed its shift in slow-regular oscillation and chaotic oscillation [9]. Neural network- (NN-) 
based modeling is the most common type. It has many advantages, such as large-scale parallel processing, the distributed storage of knowledge, and a strong self-learning capability. Since the NN technique does not require any prior knowledge of a model of the process, it is widely used in industry to estimate process variables in real time for monitoring and control [10]. Jiang et al. proposed a humanoid lower limb in the form of a parallel mechanism where the muscle was unevenly distributed by PAM [11]. Liu et al. proposed a bionic shoulder joint robot with a spherical hinge which was driven by a group of PAM [12].

To make an artificial leg have good flexibility and gait than human, an improved artificial leg is developed in this paper. In summary, this paper designs the mechanical system of an artificial leg, establishing the experimental platform of PAM and carrying out mathematical modeling. Besides, simulation of virtual prototype of artificial leg is verified, and conclusion is drawn through experimental research.

\section{Structural Design and Model Establishment}

2.1. Mechanical System Design of Artificial Legs. Aiming at the overall structure design of artificial leg, it uses PAM as the driving device of the knee joint and DC servo motor as the driving device of the hip and ankle joint. Simplified artificial leg structure model includes hip joint, knee joint, and ankle joint, each of which has one degree of freedom. The four-bar knee joint mechanism has many advantages [13]. The PAM drive can achieve flexion and extension. Figure 1 is a three-dimensional model of the knee joint. The hip joint controls the swing angle of the thigh bar through a harmonic reducer. The ankle joint is driven by a harmonic gear reducer and common spur gears to realize back bending and toe bending.

The driving mode is a sprocket type. As shown in Figure 2(a), the original and antagonist muscles are connected by chains through sprockets. The original muscle generates the motive force, the antagonist muscle generates the antagonistic force, and the chain drives the sprocket to rotate, so the joint can complete the stretching and flexing action. The output torque of this driving mode is larger, and the knee joint can meet the needs of the range of motion under load.

Considering the structure size of humanoid robot and the required moment of each joint, PAM of the model produced by FESTO is FESTO-MAS-20-200N-AA-MC. The installation position is shown in Figure 2(b). Both PAM are tilted, and the wrap angle of sprockets is more than $120^{\circ}$. The virtual prototype of the overall structure of the artificial leg is shown in Figure 3.

Figure 4 is the control system diagram of the artificial leg. The motion control card is connected with a computer through the ISA interface, and a DC servo motor is controlled by the PAMC motor driving amplifier, so as to control the motion of the hip and ankle joint. The motion control card controls the pneumatic artificial muscle inflation pressure by controlling the pressure proportional valve through the electric signal, so as to control the motion of the knee joint. During the movement of the artificial leg,

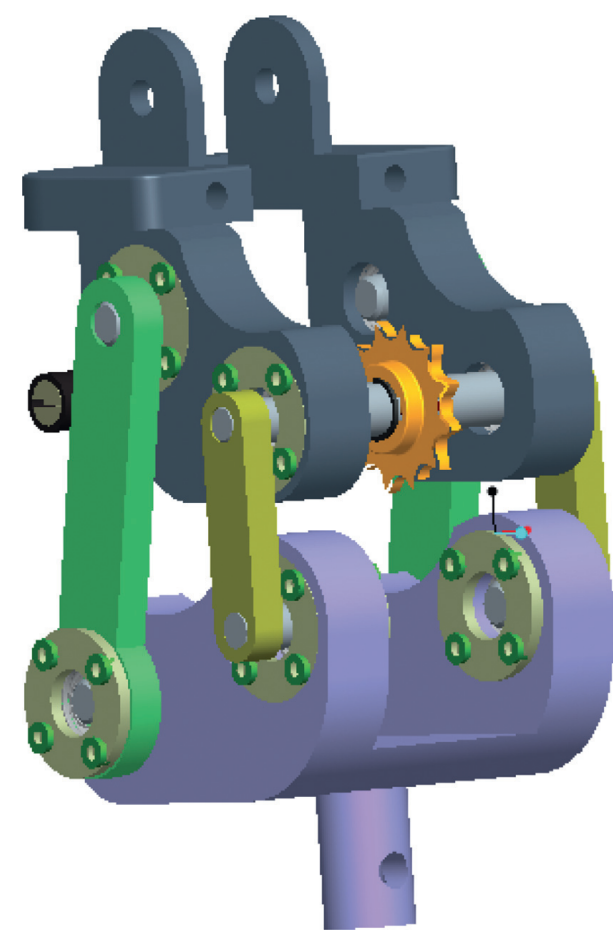

Figure 1: The structure of the four-bar knee joint.

the feedback information of the encoder and potentiometer is connected with the industrial computer through $\mathrm{D} / \mathrm{A}$.

\subsection{Construction of PAM Experimental Platform and Per-} formance Test. The static characteristic test scheme of PAM is shown in Figure 5. It is mainly composed of stepping motor, pressure proportional valve, displacement sensor, pressure sensor, tension sensor, data acquisition instrument, etc. The length of the PAM is precisely controlled by controlling the rotation of the stepping motor and using the ball screw. The pressure proportional valve controls the inflatable pressure of the pneumatic artificial muscle. Displacement sensor, pressure sensor, and tension sensor are used to measure the contraction internal pressure and output force of pneumatic artificial muscle during the experiment, respectively. The output signal is displayed by the data acquisition instrument. Figure 6 shows the actual experiment.

The mathematical model of PAM is established by means of experimental modeling. The isobaric characteristic experiment is to study the relationship between the output force and contraction rate of pneumatic artificial muscle under different pressures by loading it with the stepping motor under the condition that the input pressure of the pneumatic artificial muscle remains constant.

In the isostatic characteristic experiment, the pneumatic artificial muscle is placed horizontally. One end is connected with the tension sensor to measure the output force of the pneumatic artificial muscle during the experiment. The other end is connected with the stepping motor to apply the external force to the pneumatic artificial muscle. During the experiment, at first, the output pressure of the pressure proportional valve is adjusted so that the internal pressure of 


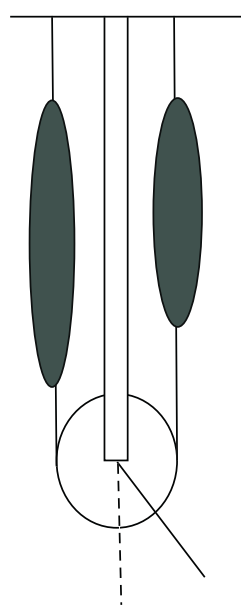

(a)

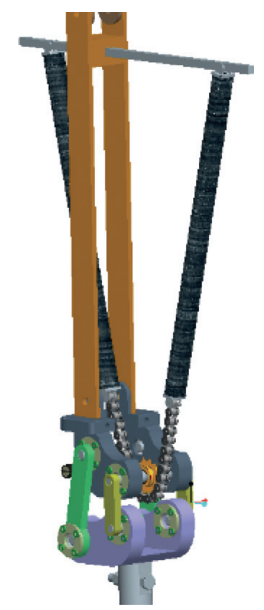

(b)

FIgure 2: Driving principle: (a) the way of joint driven by PAM and (b) the installation location of PAM.

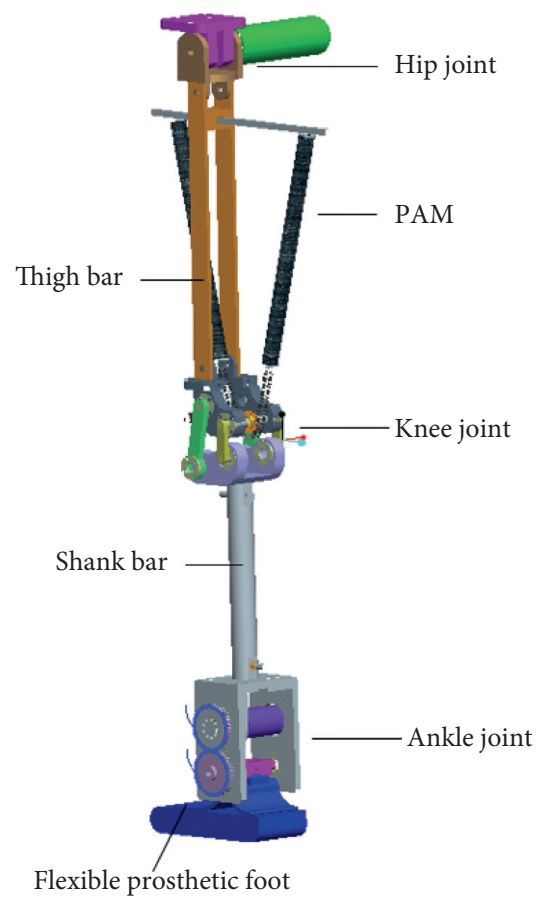

FIgUre 3: The whole structure of the artificial leg.

the pneumatic artificial muscle reaches the set value and remains fixed. At this time, the pneumatic artificial muscle is in the maximum contraction state. Secondly, the stepping motor sends a pulse signal to rotate, and the angular displacement is converted into linear displacement by the ball screw, and the pneumatic artificial muscle contracted by the inflation is gradually extended under the action of the external load. Then, stop the rotation of the stepper motor at the original length. Then, change the direction of rotation of the stepper motor so that the pneumatic artificial muscle gradually returns to the maximum contraction state, completing a work cycle. The tension sensor and the displacement sensor, respectively, measure the output force and length change of the pneumatic artificial muscle during the

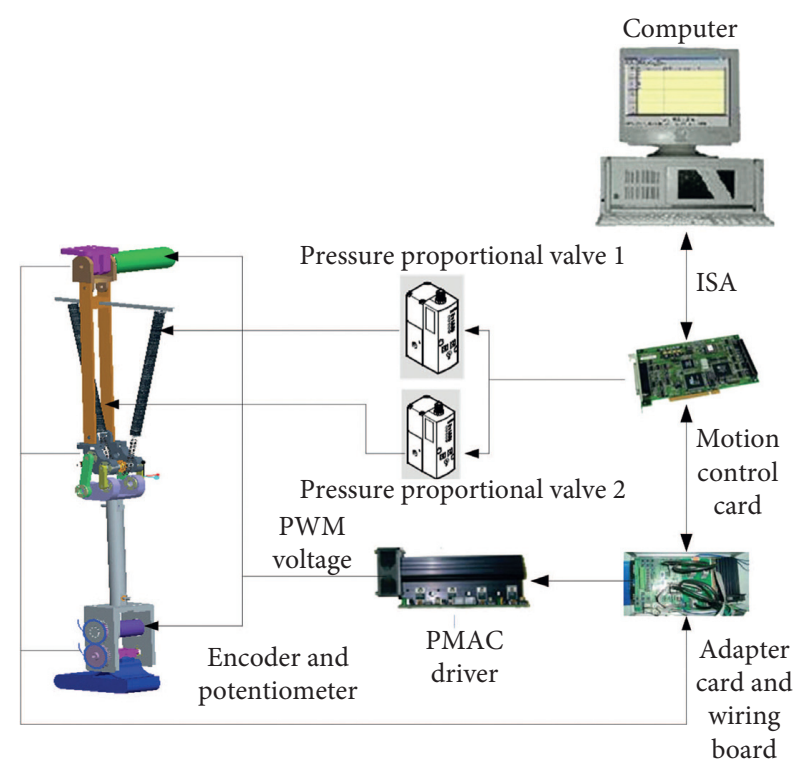

Figure 4: The control system of the artificial leg.

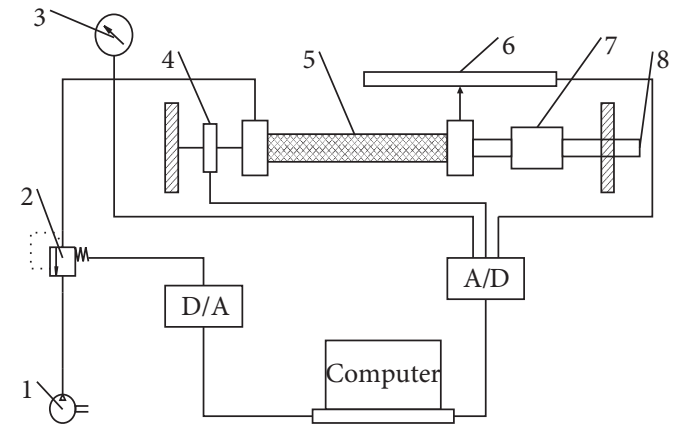

FIgURE 5: The experimental program of static characteristics of PAM. 1, air compressor; 2, pressure proportional valve; 3 , pressure sensor; 4, tension sensor; 5 , pneumatic artificial muscle; 6 , displacement sensor; 7 , stepper motor; 8 , ball screw. 


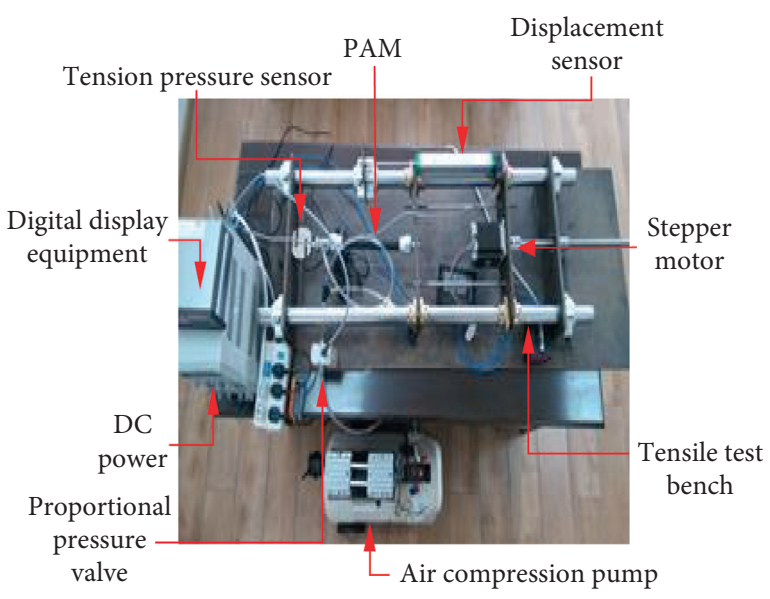

FIgURE 6: The physical diagram of experiment.

experiment, and data are recorded separately after the system is stable. Finally, the inflation pressure of the pneumatic artificial muscle is changed, and the above experimental process is repeated, and the isostatic characteristic experiment of the pneumatic artificial muscle under different pressures can be completed, thereby obtaining the contractile force and shrinkage of the PAM under different pressures.

From Figure 7, it can be seen that the internal friction of pneumatic muscle has little change under different inflating pressures. If the inflatable pressure of pneumatic artificial muscle is regarded as the activation level of biological muscle, the characteristic curve between contractile force and shrinkage of pneumatic artificial muscle is similar to biological muscle.

\subsection{Mathematical Modeling of Pneumatic Artificial Muscle.} The static model of pneumatic artificial muscle is identified by the isobaric characteristic experiment. Considering the elastic deformation of the rubber tube and the influence of friction force between the net and rubber layer on the mathematical model of pneumatic artificial muscle, the mathematical model of pneumatic artificial muscle was established by using formulae (1) and (2) by parameter identification [14].

$$
\begin{aligned}
F & =k_{0} \pi r_{0}^{2} p\left[a\left(1-k_{1} \varepsilon\right)^{2}-b\right]+k_{2}-F_{t}, \\
f_{t} & =\frac{2 \sqrt{3 a}}{3} \pi r_{0} L_{0} t E(1-\varepsilon)^{2}\left(1-\sqrt{\frac{3}{3 b-a(1-\varepsilon)^{2}}}\right),
\end{aligned}
$$

where $a=3 / \tan ^{2} \theta_{0}, b=1 / \sin ^{2} \theta_{0}, r_{0}$ is initial radius of pneumatic artificial muscle, $p$ is relative pressure in pneumatic artificial muscle inner cavity, $\varepsilon$ is contraction rate of pneumatic artificial muscle, $L_{0}$ is initial length of pneumatic artificial muscle, $\theta_{0}$ is initial angle of pneumatic artificial muscle, $t$ is wall thickness of rubber tube for pneumatic artificial muscle, and $E$ is the elastic modulus of pneumatic artificial muscle rubber tube. Assuming that the PAM is always an ideal cylinder, but in practical application, the end of the pneumatic artificial muscle will be hemispherical after inflating. The larger the contraction rate of the PAM, the smaller the effective contraction part will be, and ultimately the actual maximum contraction rate will be less than the theoretical value. To solve this problem, the physical contraction rate is converted to the effective contraction rate of the pneumatic artificial muscle by the parameter $k_{1}$. The static friction between the rubber and braided layer and fibers is not taken into account in the theoretical formula, but it is modified by parameter $k_{2}$. Considering that the abovementioned parameters will inevitably produce some errors in the theoretical formula correction, which will affect the output force of the pneumatic artificial muscle, the factors are compensated by the parameter $k_{0}$.

The least squares method is used to identify the parameters of the pneumatic artificial muscle. The estimated values of the parameters of the pneumatic artificial muscle during contraction and extension are obtained in Tables 1 and 2 .

The identified parameters are brought into the modified model. The mathematical model of the pneumatic artificial muscle during contraction and extension is obtained. The comparison with the theoretical model is made, as shown in Figure 8 , when $p=0.5 \mathrm{MPa}$.

The modified mathematical model of the pneumatic artificial muscle can accurately reflect the static characteristics when $p=0.1,0.2,0.3,0.4,0.5 \mathrm{MPa}$. In practical application, many structural parameters of the pneumatic artificial muscle are difficult to accurately measure. Therefore, the establishment of a simpler mathematical model to replace the complex modified model will be conducive for the control of pneumatic artificial muscle.

In the experiment of isobaric characteristic, considering the nonlinearity of the output force and displacement of pneumatic artificial muscle under constant pressure, the fitting function of the mathematical model of pneumatic artificial muscle is presented as follows:

$$
F=a-b \varepsilon+c \exp (-\mu \varepsilon)
$$

where $a, b$, and $c$ are fitting coefficients, $\mu$ is the nonlinear attenuation coefficient describing shrinkage, and $\mu=45$.

The fitting function of input pressure $p$ for $a(p), B(p)$, and $C(p)$ of pneumatic muscle prosthesis under contraction and stretching modes is obtained, as shown in Figure 9. On this basis, the parameter identification results of the two modes are evaluated separately, as shown in Table 3.

Finally, the mathematical model of pneumatic artificial muscle is obtained as follows:

Contraction state:

$$
\begin{aligned}
F(p, \varepsilon)= & 2442 p-197.8-(5464 p+1735) \varepsilon \\
& +(688.3 p-9.3) \exp (-45 \varepsilon)
\end{aligned}
$$

Extension state: 


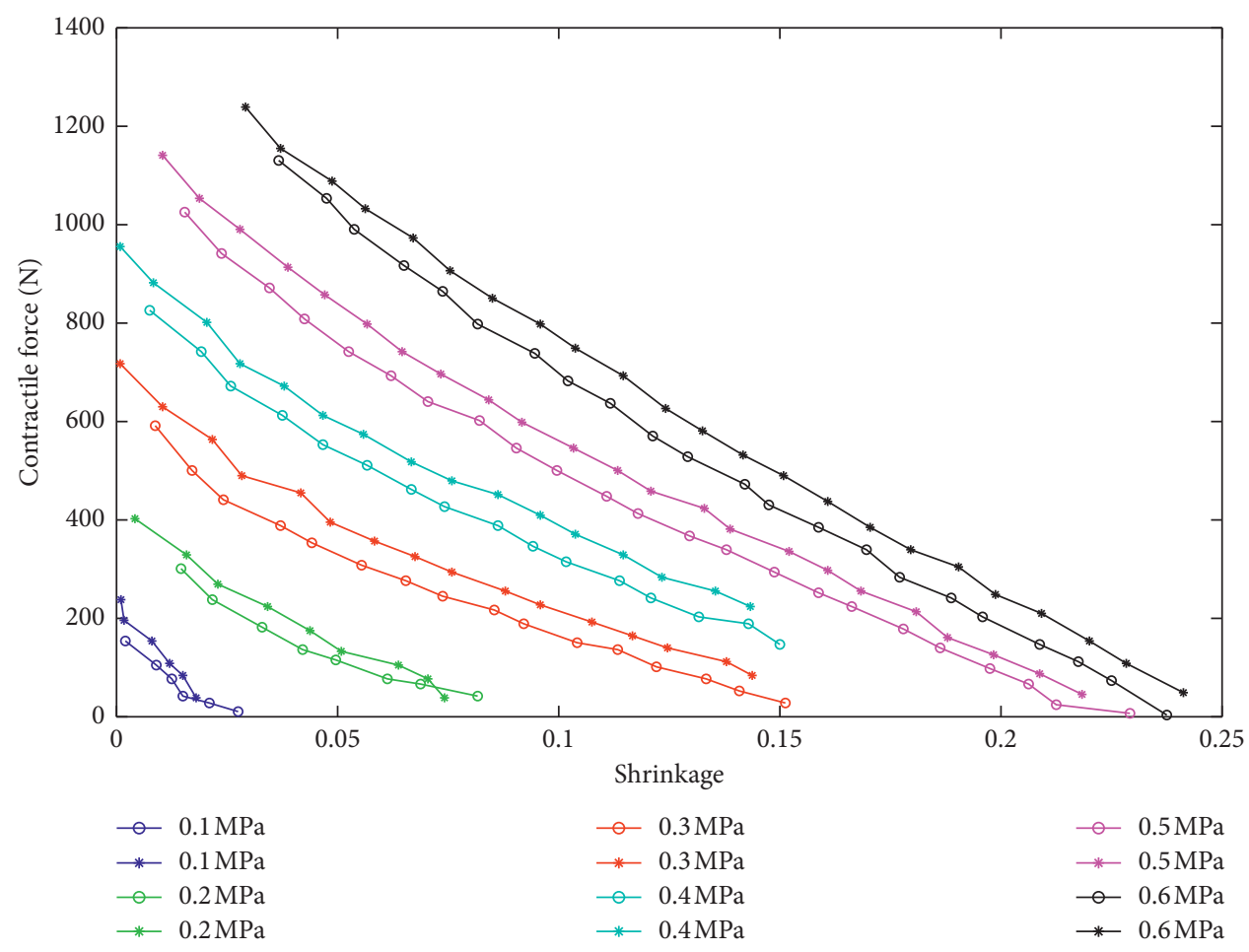

FIgURE 7: The relation of contractile force and shrinkage under the condition of equal pressure.

TABLE 1: The estimated value of the parameter with the PAM shrinking.

\begin{tabular}{lccc}
\hline Relative pressure $p(\mathrm{MPa})$ & $k_{0}$ & $k_{1}$ & $k_{2}$ \\
\hline 0.5 & 1.01 & 1.547 & 120.2 \\
0.4 & 0.8576 & 2.183 & 303.7 \\
0.3 & 0.7599 & 3.1 & 343.7 \\
0.2 & 0.7616 & 4.461 & 298.9 \\
0.1 & 0.9846 & 7.331 & 179.2 \\
\hline
\end{tabular}

TABLE 2: The estimated value of the parameter with the PAM stretching.

\begin{tabular}{lccr}
\hline Relative pressure $p(\mathrm{MPa})$ & $k_{0}$ & $k_{1}$ & $k_{2}$ \\
\hline 0.5 & 0.8466 & 1.988 & 300.2 \\
0.4 & 0.7655 & 2.433 & 326.8 \\
0.3 & 0.6977 & 3.241 & 316.3 \\
0.2 & 0.6804 & 4.676 & 266.2 \\
0.1 & 0.761 & 11.06 & 203.2 \\
\hline
\end{tabular}

$$
\begin{aligned}
F(p, \varepsilon)= & 2554 p-344.5-(8430 p-43.2) \varepsilon \\
& +(899.2 p+43.24) \exp (-45 \varepsilon) .
\end{aligned}
$$

The static mathematical model of pneumatic artificial muscle can be rewritten as follows:

$$
F(p, \varepsilon)=f_{0}(\varepsilon)+f_{1}(\varepsilon) p
$$

where

$$
\begin{aligned}
& f_{0}(\varepsilon)= \begin{cases}-197.8-1735 \varepsilon-9.3 \exp (-45 \varepsilon), & \text { constraction state, } \\
-344.5+43.2 \varepsilon+43.24 \exp (-45 \varepsilon), & \text { extension state, }\end{cases} \\
& f_{1}(\varepsilon)= \begin{cases}2442-5464 \varepsilon+688.3 \exp (-45 \varepsilon), & \text { constraction state } \\
2554-8403 \varepsilon+899.2 \exp (-45 \varepsilon), & \text { extension state. }\end{cases}
\end{aligned}
$$




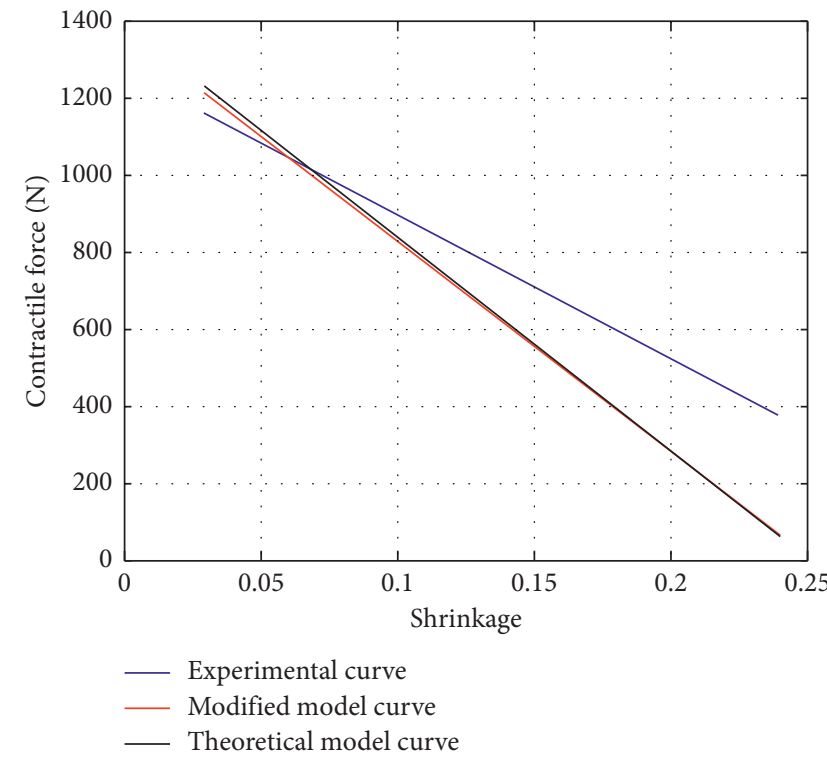

(a)

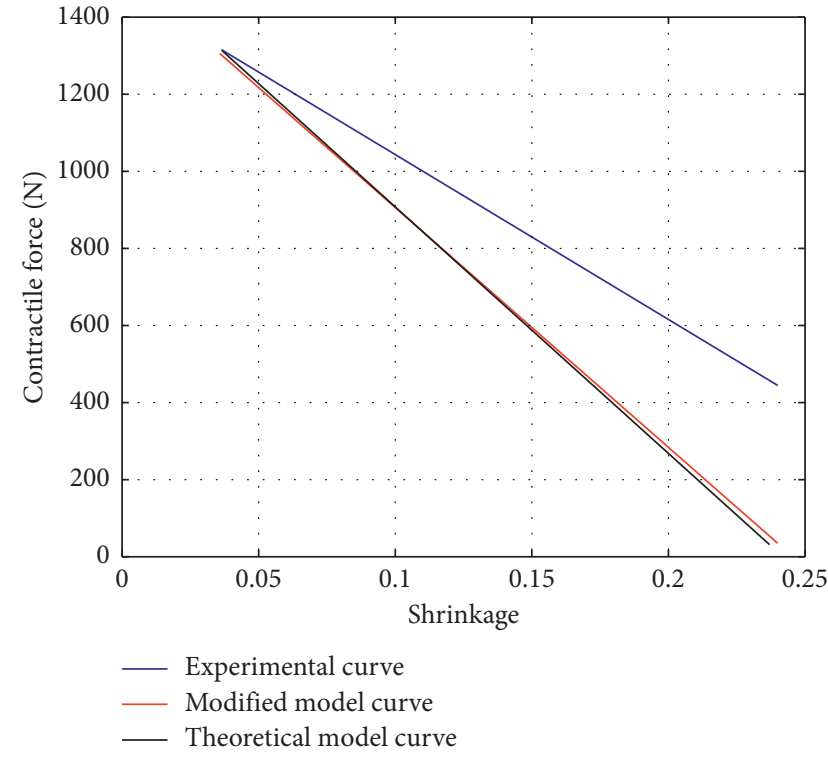

(b)

FIGURE 8: The comparison between mathematical model and experimental data with the PAM shrinking and stretching of $p=0.5 \mathrm{MPa}$.

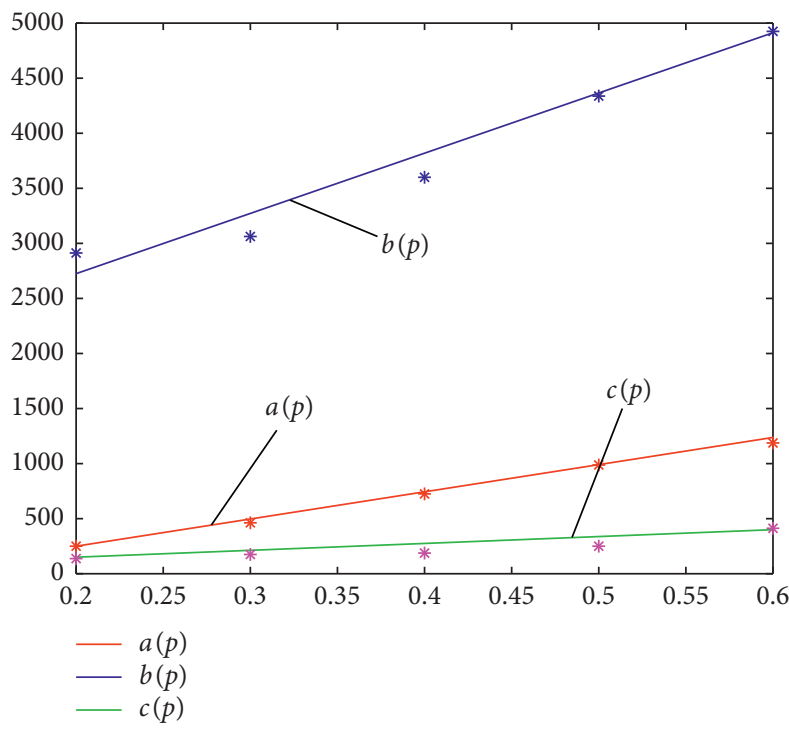

(a)

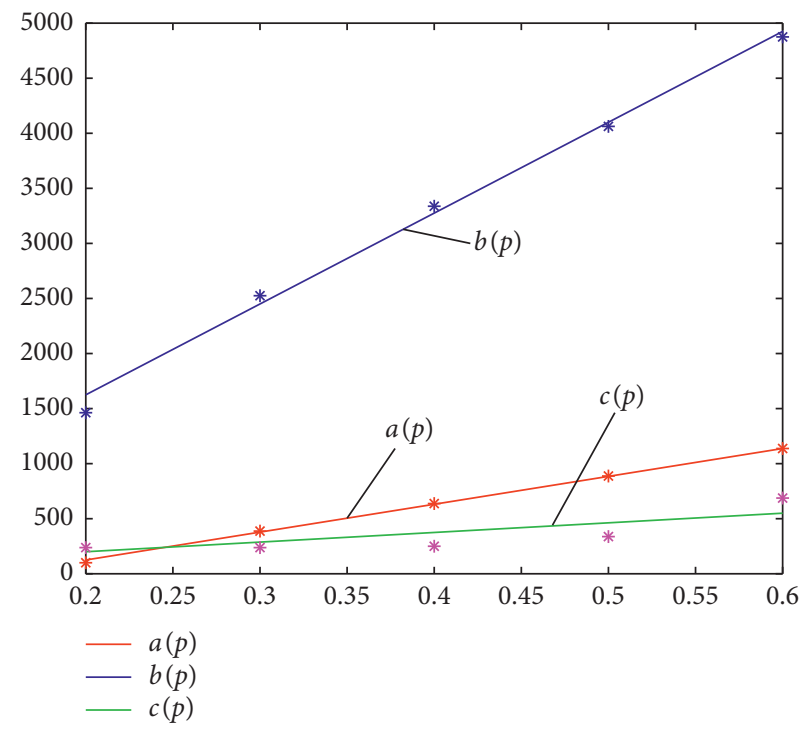

(b)

FIgURE 9: Fitting curve of $a, b$, and $c$ on pressure $p$ with the PAM shrinking (a) and stretching (b).

TABLE 3: The fitting correlation coefficient of $a, b$, and $c$ on pressure $p$ with PAM shrinking and stretching.

\begin{tabular}{lcc}
\hline Coefficient & $\rho$ (shrinking) & $\rho$ (stretching) \\
\hline$a(p)$ & 0.9997 & 0.9995 \\
$b(p)$ & 0.9647 & 0.9937 \\
$c(p)$ & 0.9758 & 0.9548 \\
\hline
\end{tabular}

As shown in Figure 10, the mathematical model can accurately reflect the static characteristics of PAM. It is relatively simple and lays a good foundation for control simulation.

\section{Experimental Simulation and Result Analysis}

3.1. Dynamic Analysis of Driving Joints. Two pneumatic artificial muscles are pulled together to drive the joint by connecting the chain and sprocket with the pneumatic artificial muscle. The principle is shown in Figure 11.

As shown in Figure 11, $F_{1}$ and $F_{2}$ are the output forces of flexor and extensor, respectively. $R$ is the force arm of the joint driven by pneumatic artificial muscle, $T$ is the driving moment, $J$ is the inertia of the load, $C$ is the damping of the joint, and $\theta$ is the rotation angle of the joint. The dynamic equation of the driving joint is established as follows: 


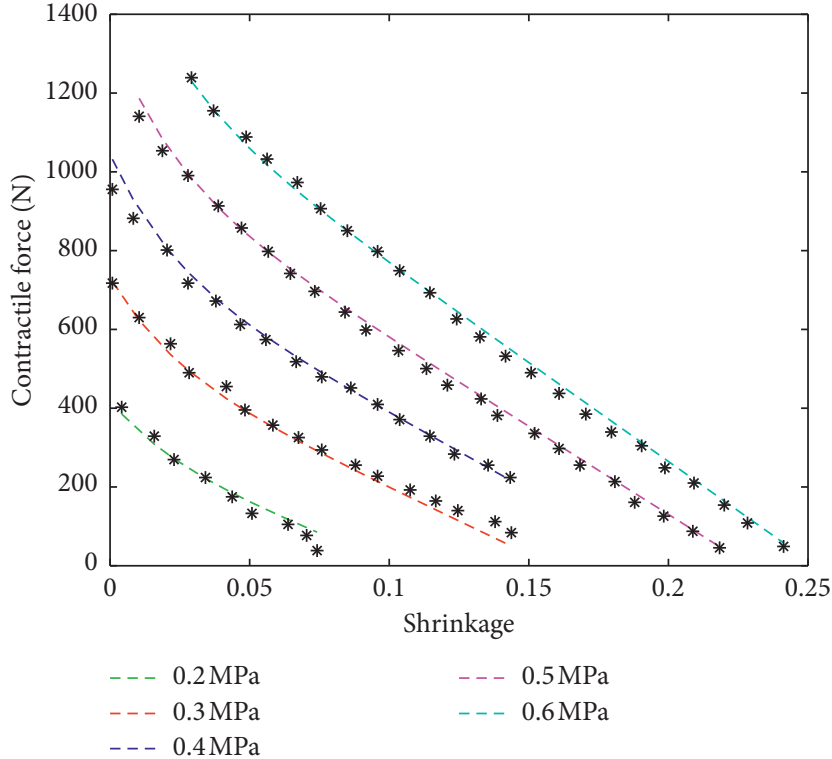

(a)

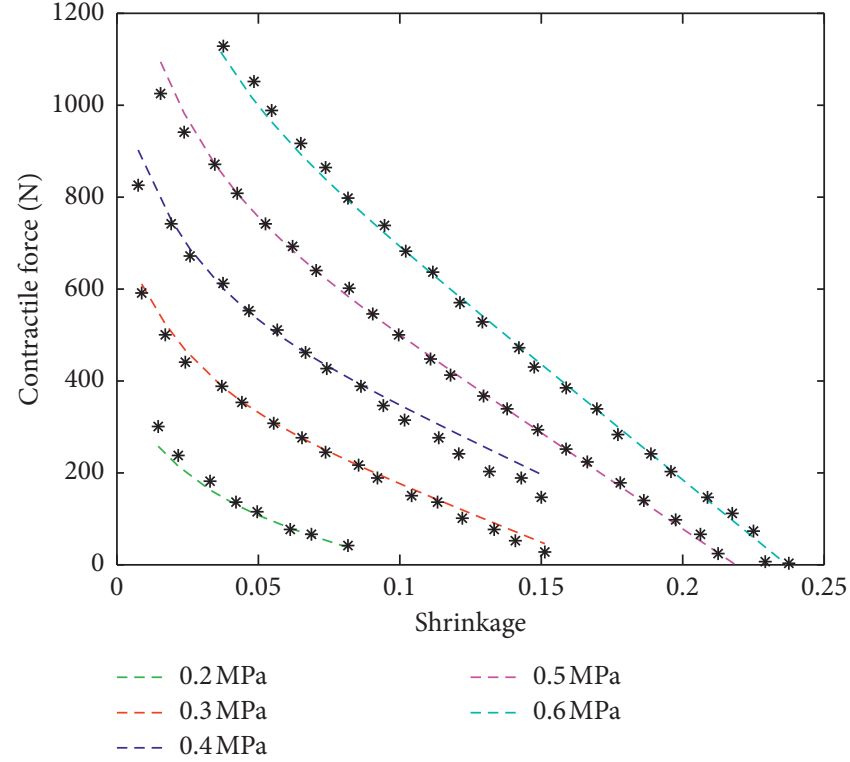

(b)

FIGURE 10: Comparison of the static mathematical model with experimental data when PAM shrinking and stretching.

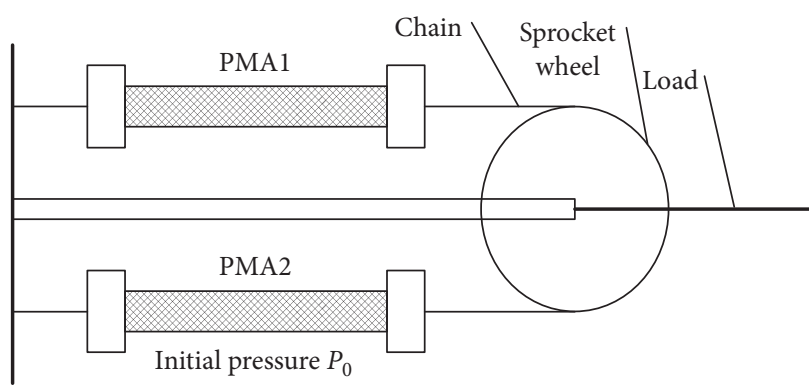

(a)

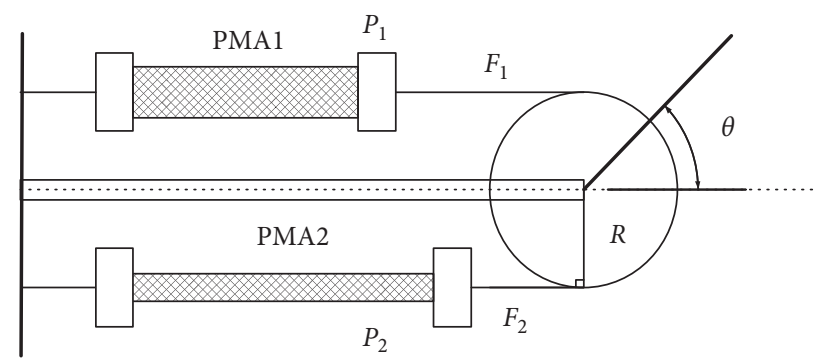

(b)

FIgURE 11: The principle of joint driven by PAM.

$$
\left\{\begin{array}{c}
J \ddot{\theta}+C \dot{\theta}=T, T=\left(F_{1}-F_{2}\right) R, \\
T=\varphi_{0}\left(\varepsilon_{1}, \varepsilon_{2}\right)+\varphi_{1}\left(\varepsilon_{1}, \varepsilon_{2}\right) \Delta p \\
\Delta p=\frac{J \ddot{\theta}+C \dot{\theta}-\varphi_{0}\left(\varepsilon_{1}, \varepsilon_{2}\right)}{\varphi_{1}\left(\varepsilon_{1}, \varepsilon_{2}\right)} .
\end{array}\right.
$$

3.2. Dynamic Model of PAM Inflation. Assuming that there is no heat exchange between the pneumatic artificial muscle and the outside during the inflating process, the process can be regarded as an adiabatic inflating process. According to the energy conservation equation of constant gas source to variable volume adiabatic charging, the energy equation of pneumatic artificial muscle charging process can be obtained [15]:

$$
\frac{\mathrm{d} p^{\prime}}{\mathrm{d} t}=\frac{\kappa \mathrm{RT}_{s} q_{m 1}}{V}-\frac{\kappa p^{\prime}}{V} \frac{\mathrm{d} V}{\mathrm{~d} t}
$$

where $q_{m 1}$ is the mass flow rate of a gas, and unit is $\mathrm{kg} / \mathrm{s} ; p^{\prime}$ is the internal pressure of an artificial muscle, and unit is $\mathrm{Pa} R$ is a gas constant, $R=287 \mathrm{~N} * \mathrm{~m} /(\mathrm{kg} * \mathrm{~K}) ; \kappa$ is isentropic index and $\kappa=1.4$; and $T_{s}$ is gas source temperature.

The mass flow equation of pneumatic artificial muscle during aeration is as follows:

$$
q_{m 1}=\frac{A_{e} p_{s}^{\prime} \sqrt{1-b^{\prime}}}{\sqrt{\mathrm{RT}_{s}}} \omega
$$

where $A_{e}$ is effective area of pipeline system, $p_{s}$ is gas source pressure, $T_{s}$ is gas source temperature, and $b^{\prime}$ is critical pressure ratio.

Finally, the dynamic model of the joint driving the artificial muscle is obtained as follows:

$$
\omega=\left\{\sqrt{1-\left(\frac{\sigma-b^{\prime}}{1-b^{\prime}}\right)^{2}}, \quad \sigma=\frac{p^{\prime}}{p_{s}^{\prime}}>b^{\prime}, 1, \sigma=\frac{p^{\prime}}{p_{s}^{\prime}} \leq b^{\prime} .\right.
$$




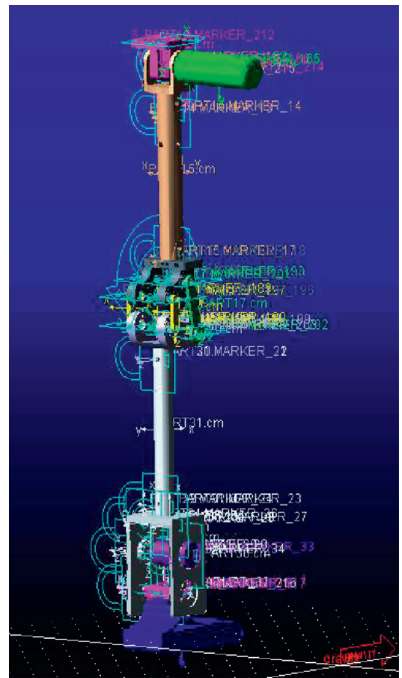

FIgURE 12: The virtual prototype of artificial leg in ADAMS.

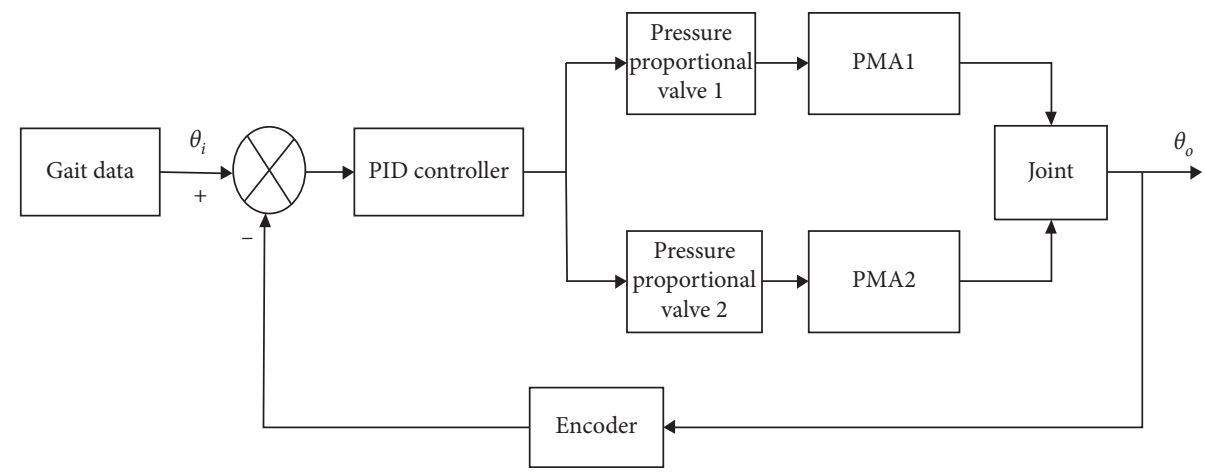

Figure 13: The process of knee joint driven by PAM.

3.3. Construction of Virtual Prototyping Joint Simulation Platform. Pro/E, ADAMS, and MATLAB/Simulink are used to build a virtual prototype joint simulation platform for artificial legs, which replace physical model to solve and control simulation. The built three-dimensional model is imported into ADAMS for dynamic analysis. The main models imported in ADAMS are parts, joint, driver, ground, and so on. Each part is connected by a pair of motion pairs. The virtual prototype model of artificial leg imported into ADAMS is shown in Figure 12, and the constraints and driving conditions of each joint are given.

The virtual prototype model of the artificial leg in ADAMS is imported into MATLAB/Simulink, and the corresponding control simulation system block diagram is built based on the PID control algorithm. The joint control simulation of the artificial leg virtual prototype is carried out, and the causes of the error between ideal gait and actual gait are analyzed. Among them, the gait data come from the human knee motion trajectory disclosed by APAS software. The sampling interval is $0.005 \mathrm{~s}$, the simulation time is $0.2 \mathrm{~s}$, and the simulation time interval is $0.005 \mathrm{~s}$.

For the knee joint, the PID control flow is shown in Figure 13. The error between ideal gait data and encoder measurement value is used as feedback signal, and the output pressure of pressure proportional valve is regulated by PID controller to regulate the output force of pneumatic artificial muscle. In practical application, one of the two pneumatic artificial muscles is inflated and contracted, and the other is deflated and stretched, so their output forces are different, which will generate driving moment on the driving shaft of the pneumatic artificial muscles, and use this moment as input of the control module.

In control simulation, the virtual prototype of artificial leg driven by pneumatic artificial muscle is generated into module my_test by ADAMS/controls, and its structure is shown in Figure 14. Control_torque is the driving moment of the knee joint, and angle_1 and angle_2 are the rotation angles of the knee joint. Taking it as the control object, and the system block diagram of control simulation is built by connecting it with other control modules in MATLAB and Simulink.

Based on the PID control algorithm, a block diagram of the control system of the pneumatic artificial muscle-driven artificial leg virtual prototype is built in MATLAB/Simulink, as shown in Figure 15. In the simulation process, the error between ideal gait trajectory and actual gait is used as feedback signal, and the input of the control module is corrected by adjusting the PID controller, which is the 

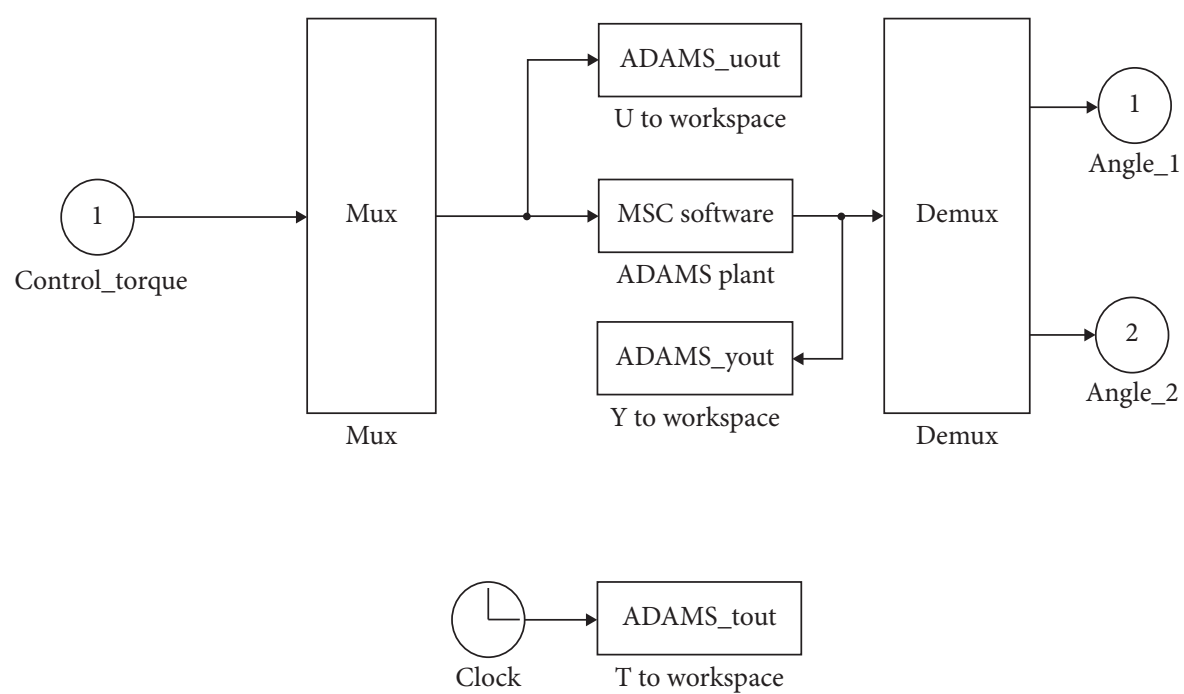

Figure 14: Control plant generated by ADAMS.

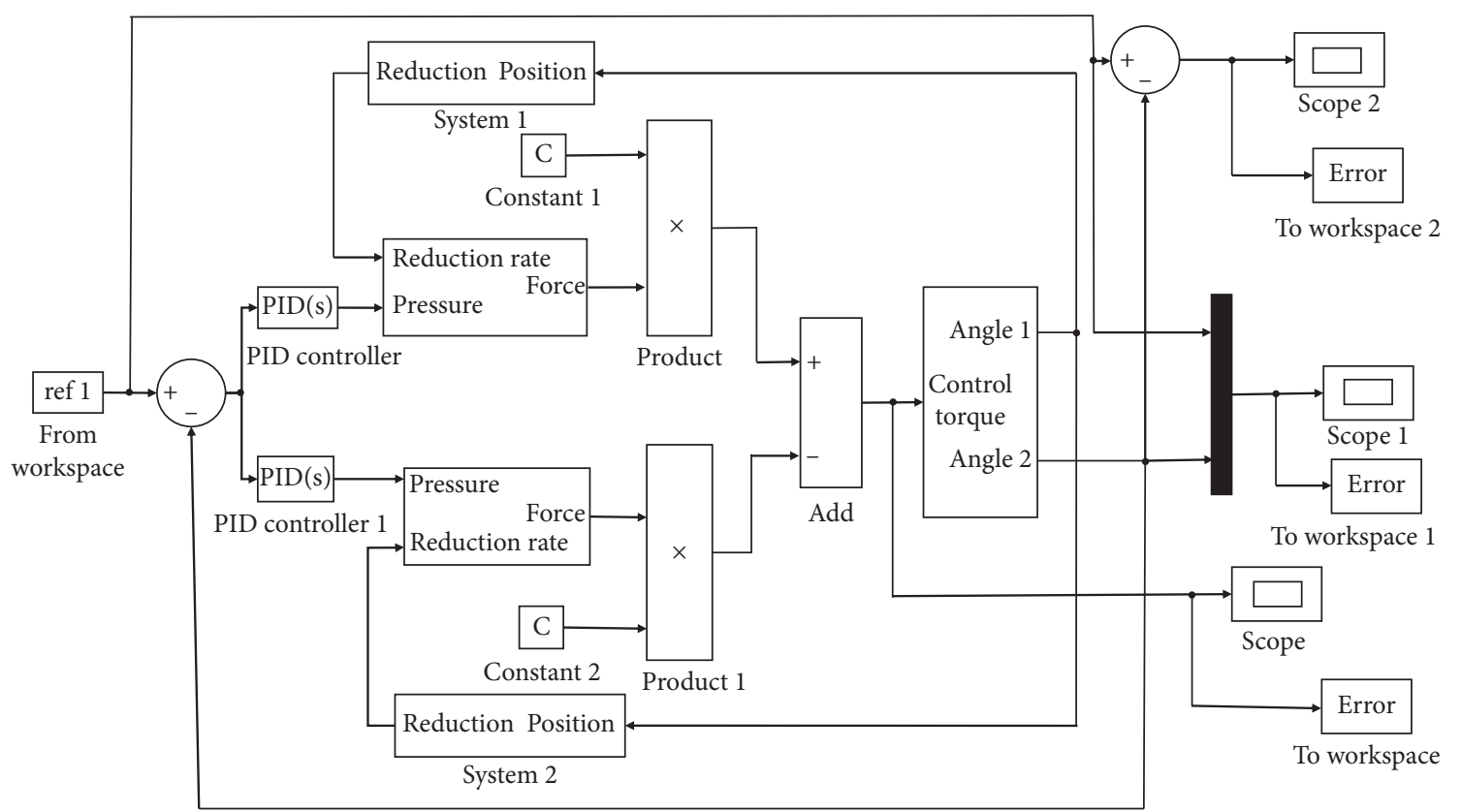

Figure 15: Simulink model of PID control algorithm.

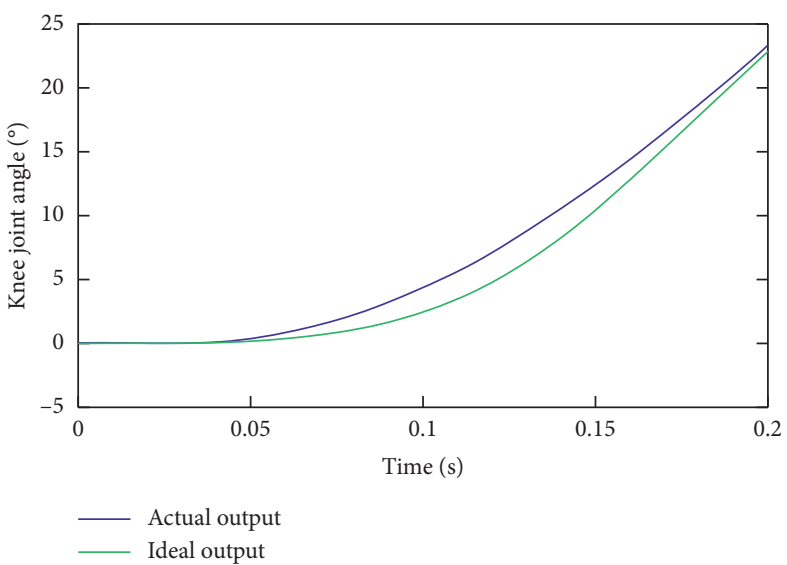

(a)

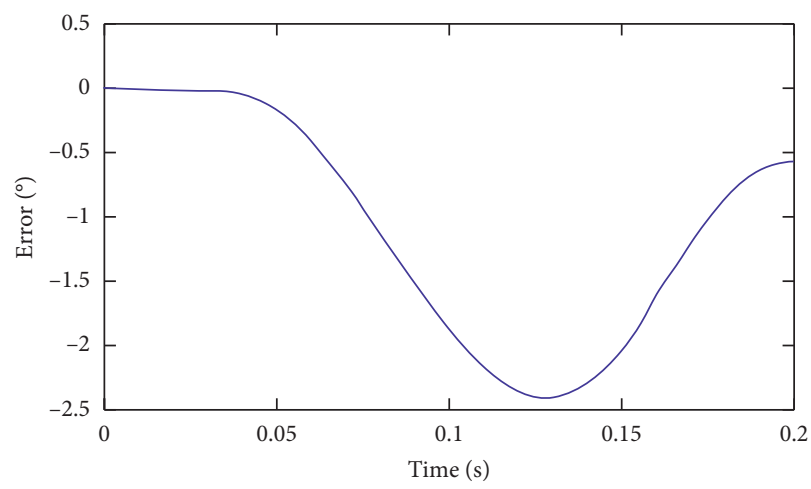

Figure 16: The tracking curve and the tracking error of knee joint. 
inflatable pressure of the pneumatic artificial muscle, and the driving moment of the knee joint is obtained. As the input of the virtual prototype model packaged by ADAMS, the rotation angle of the knee joint is obtained, which is the actual output of the control simulation system.

Using the "trial-and-error" method, the actual output curve and the ideal curve can be well fitted by many times of trial-and-error.

The tracking curve and the tracking error of knee joint as shown in Figure 16. The following steady-state error is relatively small, and the following goal is better achieved.

The causes of errors are as follows:

(1) Structural design: in the process of the structural design of the artificial leg and knee joint, although the mechanism has been optimized, it is different from the structure of the human lower limb knee joint

(2) Control algorithm: the selection of the main parameters of the PID control algorithm has a great impact on the performance of the control system, and the "trial-and-error" method is bound to have an impact on the results

(3) The influence of the nonlinearity and hysteresis of pneumatic artificial muscle on the control system

\section{Conclusion}

In this paper, the four-bar mechanism is used as the structure design of knee joint, and the structure of the artificial leg is synthetically designed. It is similar to the lower limb joint of the human body, and it can better meet the humanoid requirements of artificial leg. In terms of driving, pneumatic artificial muscles are used as the driving source of the knee joint, thereby avoiding large stiffness and other issues caused by using servo motors or hydraulic devices. An experimental platform for testing the performance of the pneumatic artificial muscle is built, which shows that the characteristic curve of the pneumatic artificial muscle is similar to biological muscle. A more accurate mathematical model is established and compared with experimental data. The results show that it can accurately reflect the static characteristics of the pneumatic artificial muscle, laying the foundation for control simulation. The dynamic analysis of the pneumatic artificial muscle driving joint is carried out, and the mathematical model of the driving joint is established. A joint simulation platform is built, and the virtual prototype of artificial leg is simulated based on the PID control algorithm. The simulation results show that the artificial leg driven by the pneumatic artificial muscle can follow the target trajectory well.

4.1. Limitations. Human lower limbs have six degrees of freedom, which are simplified to three in this paper. Better design of the artificial leg structure is required. The PID control algorithm has poor adaptability to nonlinear and time-varying systems, and better control algorithm is needed for simulation.

\section{Data Availability}

The data used to support the findings of this study are available from the corresponding author upon request.

\section{Conflicts of Interest}

The authors declare that there are no conflicts of interest regarding the publication of this paper.

\section{Acknowledgments}

This work was supported by the National Natural Science Foundation of China (grant no. 61803272) and the Fundamental Research Funds for the Central Universities of the Ministry of Education of China (grant no. N170313025). The authors are sincerely grateful for the support.

\section{References}

[1] H. Xie, K. Chen, and F. Li, "Bionic artificial leg design and control simulation based on fuzzy PID control algorithm," in Proceedings of the 5th Annual IEEE International Conference on Cyber Technology in Automation, Control and Intelligent Systems, pp. 2097-2102, IEEE, Shenyang, China, June 2015.

[2] Q. Li, X. Qin, and S. Ying, "Useness and prospect of pneumatic artificial muscles in bionic joints," Chinese Hydraulics \& Pneumatics, vol. 4, pp. 50-51, 2007.

[3] F. Li, H. Xie, and X. Xu, "Gait planning and control implementing of biped robot with heterogeneous legs," Journal of Northeastern University: Natural Science, vol. 28, pp. 10811084, 2007.

[4] G. K. Klute, J. M. Czerntecki, and B. Hannaford, "McKibben artificial muscles: pneumatic actuators with biomechanical intelligence," in Proceedings of the 1999 IEEE/ASME International Conference on Advanced Intelligent Mechatronics, pp. 221-226, IEEE, Atlanta, GA, USA, September 1999.

[5] G. K. Klute and B. Hannaford, "Fatigue characteristics of McKibben artificial muscle actuators," in Proceedings of the 1998 IEEE/RSJ International Conference on Intelligent Robots and Systems, pp. 1776-1781, IEEE, Victoria, Canada, October 1998.

[6] M. Van Damme, P. Beyl, B. Vanderborght et al., "Modeling hysteresis in pleated pneumatic artificial muscles," in Proceedings of the IEEE International Conference on Robotics, Automation and Mechatronics, pp. 471-476, IEEE, Chengdu, China, September 2008.

[7] J. Zhong, J. Fan, Y. Zhu, J. Zhao, and W. Zhai, "One nonlinear PID control to improve the control performance of a manipulator actuated by a pneumatic muscle actuator," Advances in Mechanical Engineering, vol. 6, no. ID, Article ID 172782, 2015.

[8] Y. Wang, Q. Zhang, X. H. Xiao et al., "Trajectory tracking control of the bionic joint actuated by pneumatic artificial muscle based on robust modeling," Robot, vol. 38, pp. 249256, 2016.

[9] M. La Rosa, M. I. Rabinovich, R. Huerta, H. D. I. Abarbanel, and L. Fortuna, "Slow regularization through chaotic oscillation transfer in an unidirectional chain of Hindmarsh-Rose models," Physics Letters A, vol. 266, no. 1, pp. 88-93, 2000.

[10] L. Fortuna, P. Giannone, S. Graziani, and M. G. Xibilia, "Virtual instruments based on stacked neural networks to improve product quality monitoring in a refinery," IEEE 
Transactions on Instrumentation and Measurement, vol. 56, no. 1, pp. 95-101, 2007.

[11] F. Jiang, G. Tao, and Q. Li, "Analysis and control of a parallel lower limb based on pneumatic artificial muscles," Advances in Mechanical Engineering, vol. 9, pp. 1-14, 2017.

[12] Y. Liu, T. Wang, W. Fan et al., "Statics analysis of a bionic shoulder joint robot," Transactions of Beijing Institute of Technology, vol. 35, pp. 607-611, 2015.

[13] X. Wu, S. Zhai, and Q. Hao, "Optimization and simulation of the four-bar prosthetic knee joint," Machinery Design, vol. 28, pp. 42-45, 2011.

[14] P. Chou and B. Hannaford, "Measurement and modeling of Mckibben pneumatic artificial muscles," IEEE Transactions on Robotics and Automatic, vol. 12, no. 1, pp. 90-102, 1996.

[15] J. Li, Dynamics of Pneumatic Transmission System, South China University of Technology Press, Guangzhou, China, 1991. 